\title{
Development of a Novel Bioreactor for the Mechanical Loading of Tissue-Engineered Heart Muscle
}

\author{
R.K. BIRLA, Ph.D., ${ }^{1}$ Y.C. HUANG, Ph.D., ${ }^{2}$ and R.G. DENNIS, Ph.D. ${ }^{3}$
}

\begin{abstract}
Objective: In this study, we describe a novel bioreactor system to deliver controlled stretch protocols to bioengineered heart muscle (BEHM) constructs. Our primary objective was to evaluate the effect of mechanical stretch on the contractile properties of three-dimensional cardiac constructs in vitro. Methods: BEHMs were formed by culturing primary neonatal cardiac myocytes in a fibrin gel using a method previously developed in our laboratory. A custom bioreactor system was designed using SolidWorks (Concord, MA) and structural components were manufactured using fusion deposition modeling. We utilized the bioreactor to evaluate the effect of 2-, 6-, and 24-hour stretch protocols on the stretch-induced changes in contractile function of BEHMs. Results: We were able to demonstrate compatibility of the bioreactor system with BEHMs and were able to stretch all the constructs with zero incidence of failure. We found that loading the constructs for 2, 6, and 24 hours during a 24-hour period using a stretch protocol of $1 \mathrm{~Hz}, 10 \%$ stretch did not result in any significant change in the active force, specific force, pacing characteristics, and morphological features. Conclusions: In this study, we demonstrate compatibility of a novel bioreactor system with BEHMs and the stability of the BEHMs in response to stretch protocols.
\end{abstract}

\section{INTRODUCTION}

$\mathbf{R}$ ESEARCH IN THE FIELD OF cardiac tissue engineering is focused on the development of functional threedimensional heart muscle in vitro. Conceptually, autologous patient-derived stem cells can be differentiated to a cardiac origin and utilized to engineer functional three-dimensional heart muscle. Heart muscle that has been engineered in vitro can be utilized clinically to treat cases of acute myocardial infarction. In addition, functional heart muscle would prove to be a valuable model for basic cardiology research and to evaluate the efficacy and safety of potential cardio-active drugs.

Several strategies have been described to promote the formation of three-dimensional heart muscle. Eschenhagen et al. utilized a collagen matrix to promote the culture of chick cardiac myocytes resulting in the formation of functional three-dimensional heart muscle. ${ }^{1}$ The initial model was refined by casting a mixture of neonatal cardiac myocytes and collagen into plastic molds to generate engineered heart tissue. ${ }^{2}$ Carrier et al. utilized fibrous meshes made from polyglycolic acid as a scaffolding material for culturing neonatal cardiac myocytes. ${ }^{3}$ Okano and coworkers utilized temperature-sensitive polymer surfaces to engineer three-dimensional cardiac tissue. ${ }^{4,5}$ Akins et al. utilized a rotating bioreactor system to promote the organization of isolated cardiac cells on the surface of microcarrier beads. ${ }^{6}$ Collectively, the work supports the ability of primary cardiac myocytes to form tissue-engineered heart muscle under controlled in vitro conditions.

\footnotetext{
${ }^{1}$ Section of Cardiac Surgery, University of Michigan, Ann Arbor, Michigan.

${ }^{2}$ Department of Biomedical Engineering, University of Michigan, Ann Arbor, Michigan.

${ }^{3}$ Department of Biomedical Engineering, University of North Carolina, Chapel Hill, North Carolina.
} 
We have previously developed a method to engineer functional three-dimensional skeletal ${ }^{7-9}$ (myooids) and cardiac ${ }^{10}$ (cardioids) muscles in vitro. In addition, we have recently extended our cardioid model and shown the utility of using fibrin gel to support the formation of bioengineered heart muscle (BEHM). ${ }^{11}$ During BEHM formation, primary cardiac myocytes are isolated from neonatal rat hearts and plated on the surface of a tissue culture plate coated with fibrin. Spontaneous contractions of the primary cardiac myocytes result in compaction of the fibrin gel promoting BEHM formation. BEHMs are $12 \mathrm{~mm}$ long and have a diameter of 200-250 $\mu \mathrm{m}$. BEHMs have been shown to generate an active force of $300-500 \mu \mathrm{N}$ and a specific force of $12-15 \mathrm{KN} / \mathrm{m}^{2}$, and can be electrically paced at frequencies $1-7 \mathrm{~Hz}$. In addition, BEHMs are responsive to external calcium and cardio-active drugs like isoproterenol and epinephrine, and express several cardiac-specific proteins (collagen type I, F-actin, connexin43, and N-cadherin). ${ }^{11}$

Our current research is focused on defining culture conditions to promote the phenotypic maturation of BEHMs in the form of chemical, electrical, and mechanical stimulation. To this end, we have developed a new bioreactor system to allow application of coordinated electromechanical stretch to BEHMs during culture. Our bioreactor system is designed to permit coordinated, simultaneous electrical and mechanical stimulation of the BEHMs in culture. The stimulation protocols can be carried out using automated stimulation algorithms embedded in the onboard microprocessors of each bioreactor for long periods of time (weeks or months) while permitting the culture media to be changed manually, as would occur in traditional cell culture using Petri dishes.

There have been a few studies evaluating the effect of mechanical loading on tissue-engineered heart muscle. ${ }^{12,13}$ Akhyari et al. showed that a $20 \%$ strain at a frequency of $1.33 \mathrm{~Hz}$ for a period of 14 days resulted in an increase in cell proliferation and uniform cell distribution throughout the construct. ${ }^{12}$ Fink et al. showed that a stretch protocol of $20 \%$ strain at $1.5 \mathrm{~Hz}$ frequency for 6 days resulted in doubling of the active force of their cardiac constructs. ${ }^{13}$

Although there have been studies showing the effect of mechanical stretch on the functional performance of tissueengineered heart muscle, there has been a lack of adequate instrumentation to control the stimulation protocol thereby permitting variable tissue responses allowing detailed understanding of the underlying mechanism. The system developed by Fink et al. allowed a single frequency of $1.5 \mathrm{~Hz}$ to be tested, whereas the system developed by Akhyari et al. failed to correlate the stretch to contractile performance. We believe that there is a strong need to develop accurate instrumentation to carefully control tissue-stimulation parameters and correlate the degree of stretch to changes in contractile performance. To accommodate the need for instrumentation, the purpose of this study was to evaluate the feasibility of utilizing the current bioreactor to support the viability of BEHMs and to evaluate subsequent changes in contractile performance.

\section{MATERIALS AND METHODS}

Approval for animal use was granted by the University Committee for the Use and Care of Animals (UCUCA) in accordance with the "Guide for the Care and Use of Laboratory Animals" (NIH publication 86-23, 1986). All materials were purchased from Sigma (St. Louis, MO) unless otherwise specified.

\section{Bioreactor system}

The mechanical components of the bioreactor system are shown schematically in Figure 1. The system was designed using SolidWorks solid modeling and design software (Solid Works, Concord, MA). Individual structural components were rapid manufactured using a Titan model $\mathrm{T} 1$ fusion deposition modeling (FDM) machine (StrataSys, Minneapolis, $\mathrm{MN})$. The individual structural components of the bioreactor were first designed in SolidWorks, mechanical assembly and fit was verified in solid assembly drawings, and then

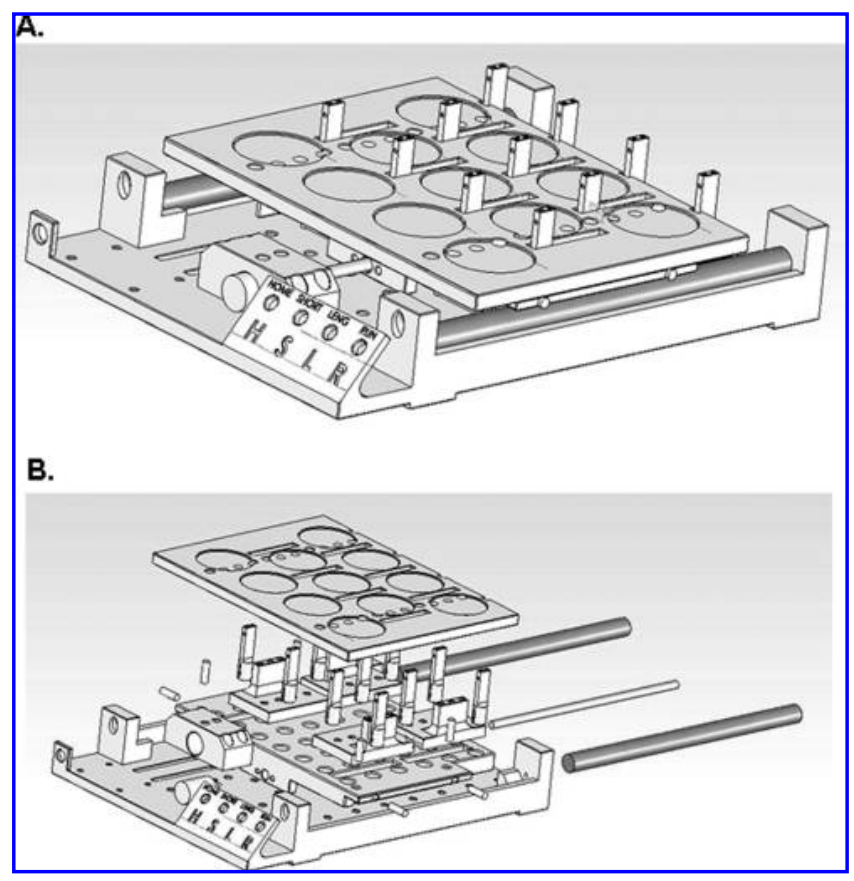

FIG. 1. Bioreactor design. (A) Primary structural components for the bioreactors were rapid manufactured from FDA-compliant polycarbonate thermoplastic using a StrataSys Titan T1 fusion deposition modeler. (B) An exploded view of the assembly. Each bioreactor also included standard commercially available off-theshelf (COTS) components, including a gearhead stepper motor (MicroMo), an ACME power screw and low-friction nut, and stainless steel type 18-8 threaded fasteners (not shown). The modifications to the COTS components were very minimal, typically limited to simple processes such as cutting stainless steel tubing to the desired length. The authors will freely provide the solid model files and a list of other components to interested researchers upon request. 
individual component files were converted to *.STL format and were rapid manufactured from U.S. Food and Drug Administration (FDA)-compliant polycarbonate (PC-10) material on the FDM. This process allowed both rapid development time and rapid manufacture of several bioreactor units for use in this study. Metal fasteners and mechanism components were purchased as commercially available offthe-shelf (COTS) mechanism components from McMasterCarr Supply (Cleveland, OH). The system was controlled by a low-cost, small form factor $(\sim 3 \times 5 \mathrm{~cm})$ stepper motor driver circuit specifically designed for this application (Fig. 2). The stepper controller circuit also allowed for direct user interface and control of each bioreactor, both for system set-up and to initiate the experimental protocol. The actual mechanical strain protocol was programmed directly onto the embedded microcontroller prior to each experiment, and was not adjustable by the user during the course of each experiment. An identical circuit can be employed to provide two channels of bipolar square-wave electrical stimulation to the tissues in culture, allowing the muscle to be activated at a known time with respect to the application of mechanical strain.

\section{General description of the system}

Each bioreactor can be loaded with up to 11 separate $35 \mathrm{~mm}$ culture dishes, each containing a single tissue construct, in this case BEHMs. The bioreactor systems are designed to readily accommodate a range of self-organizing tissues, including skeletal, cardiac, and smooth muscles, tendon and ligament, and bone. Adjacent to each $35 \mathrm{~mm}$ plate is a movable post. One end of each tissue specimen is affixed to this post via a stainless steel wire that is shaped to allow it to pass under the cover of the $35 \mathrm{~mm}$ dish and attach to the stainless steel minutien pins that affix the ends of each tissue specimen. The $35 \mathrm{~mm}$ plates do not require modification; the lids are simply tipped forward to accommodate the stainless steel wire. The culture plates are held stationary above a moving platform to which the movable posts are affixed below. Thus, during mechanical movement of the posts, the tissue specimen length is changed but the base of the culture dish remains motionless. This arrangement minimizes sloshing of the culture media that would occur if the culture dish was moved cyclically or dimensionally deformed.

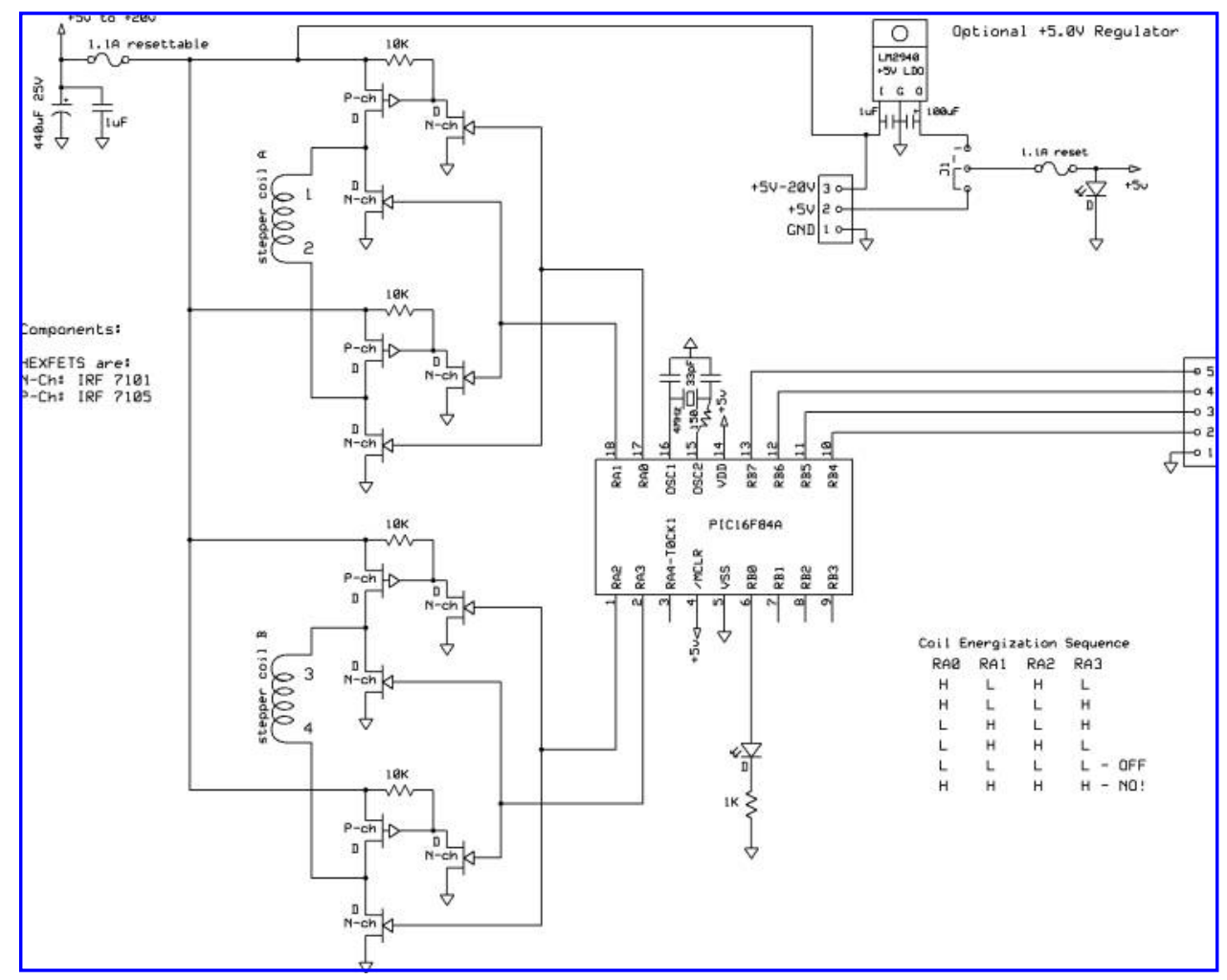

FIG. 2. Schematic diagrams for the stepper motor driver and controller. Small $(3 \times 5 \mathrm{~cm})$, low-cost stepper motor drivers were designed and manufactured using printed circuit boards designed with free software (ExpressPCB) and ordered online from ExpressPCB (www.expresspcb.com/). The embedded microcontroller (PIC16F84A) was programmed using a PIC-C compiler (CCS, Brookfield, WI) to simply energize the coils as indicated on the schematic at preselected time intervals to achieve displacement, direction, and strain-rate control for applying mechanical strain to the tissue specimens. Four bits (input registers RB4-RB7) were used to control each system by detecting the state of four momentary-ON push buttons. The four commands were (i) $<\mathrm{HOME}>$, which sends linear slide to the HOME position for setup; (ii) $<$ SHORTEN $>$ and (iii) $<$ LENGTHEN $>$, which allow manual control of the linear slide in each direction; and (iv) $<$ RUN $>$, which executes the strain protocol continuously until another command is received. 
The mechanical strain mechanism is comprised of a linear actuator that drives the moving platform beneath the culture plates. The linear actuator is a direct-drive ACME miniature power screw (ACME diameter 0.216", 4 starts, 5.2 revolutions/inch, McMaster-Carr, catalog \# 6642K16) affixed to the output shaft of the gearhead stepper motor.

Mechanical strain of the same amplitude is applied to all specimens simultaneously. In general, different mechanical stimulus protocols would be carried out on separate but identical bioreactors. Mechanical strain is controlled by a gearhead stepper motor (MicroMo Electronics, Clearwater, FL), which is driven by an open-loop stepper motor driver shown in Figure 2. The stepper motor driver is a modified version of an implantable muscle stimulator used by our laboratory, which was reported earlier. ${ }^{14}$

For each mechanical strain cycle, the stepper motor driver starts at a HOME position defined mechanically by the position of an optical interrupter (Panasonic \# ON1111 Photo Interrupter; Matsushita Electric Industrial, Osaka, Japan), which provides a single-bit digital signal to the motor driver circuit. The stepper motor driver then drives the strain mechanism at the desired rate in the desired direction for the preprogrammed length of each strain application, after which the mechanism returns to the HOME position. By this means, cycle-to-cycle mechanical drift is eliminated without the need to employ sophisticated closed-loop control. At the end of the system design and fabrication phase, the system was tested for 3 weeks with continuous cyclic strain, with no detectable drift from the original strain parameters.

\section{Isolation of neonatal cardiac myocytes}

Cardiac myocytes were isolated from the hearts of 2- to 3-day-old F344 rats using an established method. ${ }^{15}$ Briefly, hearts were cut into fine pieces and suspended in a dissociation solution that consisted of $0.32 \mathrm{mg} / \mathrm{mL}$ collagenase type II (Worthington Biochemical, Lakewood, NJ) and $0.6 \mathrm{mg} / \mathrm{mL}$ pancreatin dissolved in a buffer consisting of $116 \mathrm{mM}$ sodium chloride $(\mathrm{NaCl}), 20 \mathrm{mM}$ HEPES, $1 \mathrm{mM}$ disodium phosphate $\left(\mathrm{Na}_{2} \mathrm{HPO}_{4}\right), 5.5 \mathrm{mM}$ glucose, $5.4 \mathrm{mM}$ potassium chloride $(\mathrm{KCl})$, and $0.8 \mathrm{mM}$ magnesium sulfate $\left(\mathrm{MgSO}_{4}\right)$. Serial digestion was carried out in an orbital shaker for 5 minutes at $37^{\circ} \mathrm{C}$, and the supernatant was collected in $5 \mathrm{~mL}$ of horse serum (Invitrogen, Auckland, New Zealand). Cells from all the digests were pooled, centrifuged, and then suspended in culture medium (CM) consisting of $320 \mathrm{~mL}$ M199, $100 \mathrm{~mL}$ F12K, $50 \mathrm{~mL}$ fetal bovine serum, $25 \mathrm{~mL}$ horse serum, $5 \mathrm{~mL}$ antibiotic-antimycotic, $40 \mathrm{ng} / \mathrm{mL}$ hydrocortisone, and $40 \mu \mathrm{g} / \mathrm{mL}$ ascorbic acid (Invitrogen). The final cell concentration was adjusted to $1 \times 10^{6}$ cells $/ \mathrm{mL}$.

\section{Preparation of the plates}

The method for preparing the culture surface for engineering skeletal muscle has been described in detail previously. ${ }^{7,16}$ This procedure was modified to engineer cardiac muscle. ${ }^{10}$
Briefly, $35 \mathrm{~mm}$ culture plates were coated with $1.5 \mathrm{~mL}$ of a polydimethylsiloxane (PDMS) elastomer (Dow Chemical, Midland, MI). Anchor points were 6-mm-long segments of size 0 braided silk sutures (Ethicon, Cornelia, GA) pinned $12 \mathrm{~mm}$ apart in the center of the culture surface (Fig. 3).

\section{Formation of fibrin gel and cell plating}

To promote gel formation, $0.5 \mathrm{~mL}$ of $\mathrm{CM}$ containing $10 \mathrm{U} / \mathrm{mL}$ thrombin was plated on the surface of $35 \mathrm{~mm}$ cell culture plates coated with Sylgard (PDMS, type 184 silicone elastomer). After this, $200 \mu \mathrm{L}$ of $20 \mathrm{mg} / \mathrm{mL}$ fibrinogen was added to the $35 \mathrm{~mm}$ plate. The solution was mixed to promote the formation of a fibrin gel within 10-15 minutes. Primary cardiac cells were diluted in CM at 1 million cells/ $\mathrm{mL}$ and plated in $1 \mathrm{~mL}$ of $\mathrm{CM}$ for each plate after complete gel formation.

The cells were cultured in an incubator at $37^{\circ} \mathrm{C}$ and $5 \%$ carbon dioxide with medium changes everyday. All culture plates were maintained for 2 weeks until most fibrin was degraded, and then the mechanical-loading group was placed into the bioreactor for another 7 days. The control group was still in static culture for another 7 days.

\section{Placement of BEHMs onto the bioreactor}

The BEHMs were cultured in individual $35 \mathrm{~mm}$ culture plates until the self-organization of the BEHM was complete. After BEHM formation, the entire culture plate for each specimen was loaded onto a bioreactor as described above (Fig. 4A). First, the $35 \mathrm{~mm}$ plate was placed into a well in the bioreactor, and then an L-shaped arm composed of a stainless steel stick with L-shaped silicone tube at one end was affixed to the bioreactor. The silicone tube was kept inside the $35 \mathrm{~mm}$ plate (Fig. 4B). One stainless steel pin at the end closer to the L-shaped arm was released while another pin was connected to the silicone part by lifting the pin from the sylgard surface (Fig. 4C).

\section{Electromechanical stimulation protocol}

For our first series of experiments, we loaded the fibrin gel constructs onto the bioreactor and stimulated for 2 hours per day, 6 hours per day, and 24 hours for 7 days at a frequency of $1 \mathrm{~Hz}$ with $10 \%$ stretch. Controls were maintained in static cell culture for the 7-day period. For all studies (except the 2hour loading protocol), the active force of the BEHMs was evaluated before and after the 7-day loading protocol.

\section{Evaluation of contractility}

The method for evaluating the contractility of engineered skeletal muscle has been described in detail elsewhere. ${ }^{7}$ The method was modified to test engineered cardiac tissue. Briefly, the BEHMs were stimulated between parallel platinum electrodes, and the active force was measured using a custom-built optical force transducer. ${ }^{7}$ An electrical impulse 

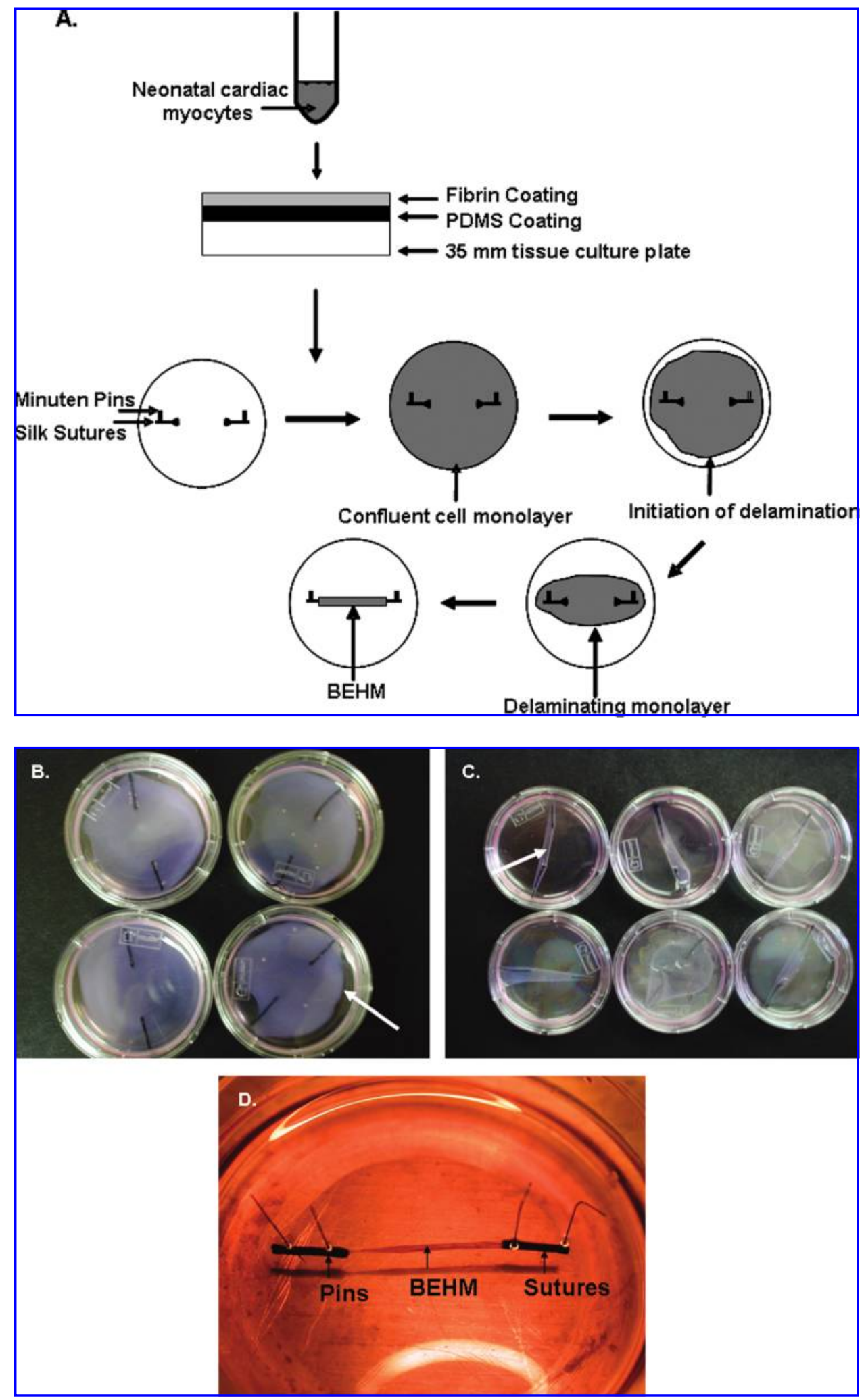

FIG. 3. Description of bioengineered heart muscles (BEHMs). (A) Methodology for construct formation: neonatal cardiac myocytes are mixed with fibrinogen and then thrombin. The cell suspension is then plated on the polydimethylsiloxane surface. The spontaneous contractility of the neonatal cardiac myocytes resulted in delamination of the cell monolayer. (B-D) Stages of BEHM formation: delamination initiated at the (B) periphery of the culture surface and progresses toward the (C) center of the culture surface. (D) After complete formation, the BEHM remained suspended between anchor points that have been pinned to the culture surface with $0.1 \mathrm{~mm}$ pins. Color images available online at www.liebertpub.com/ten. 

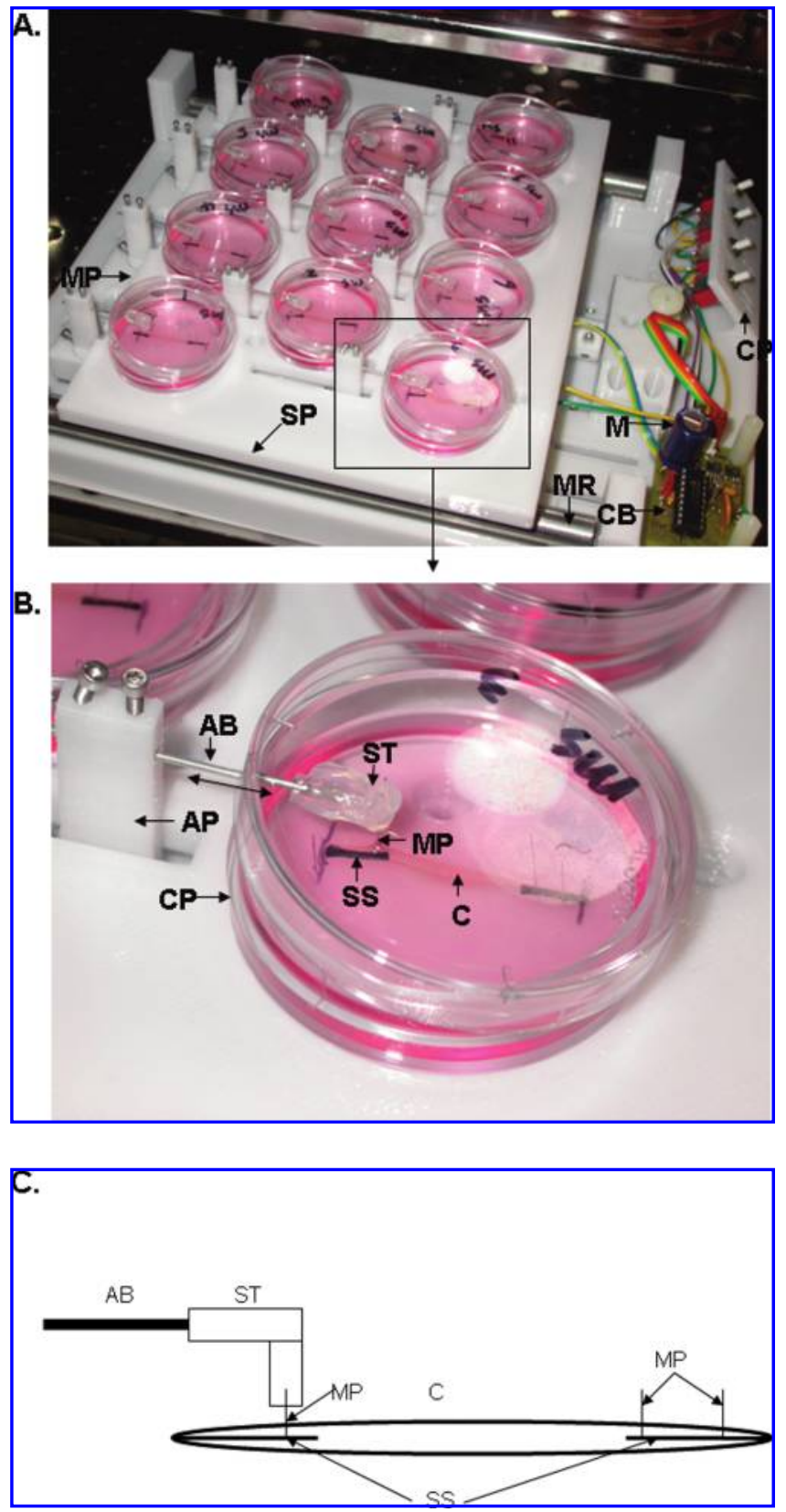

FIG. 4. The setup of the bioreactor for the cardiac constructs. (A) The overview of eleven $35 \mathrm{~mm}$ culture plates placed in the bioreactor. CP: control panel, MR: metal rod, SP: stationary platform, M: motor, CB: circuit board. (B) A close picture showing the connection between the bioreactor and bioengineered heart muscle (BEHM). One end of the BEHM had been lifted and connected to the steel arm through the steel pin and silicon tube. (C) Schematic showing the BEHM attachment to the bioreactor arm. AB: steel arm, AP: plastic support, C: construct, CP: culture plate, MP: $1 \mathrm{~mm}$ steel pin, SS: silk suture, ST: silicon tube. Color images available online at www.liebertpub.com/ten. of $15 \mathrm{~V}$ with a pulse width of $10 \mathrm{~ms}$ and a frequency of $1 \mathrm{~Hz}$ was used. The active force was measured using a custommade optical force transducer, and force tracings were digitally recorded using LabVIEW (National Instruments, Austin, TX). The active force was normalized to the total cross-sectional area of the BEHM to obtain the specific force. The cross-sectional area of the BEHMs was calculated from the construct diameter, which was determined by a calibrated eyepiece reticle with a resolution of $5 \mu \mathrm{m}$.

\section{Histology}

BEHMs were fixed in a $4 \%$ paraformaldehyde solution for 4 hours, rinsed in $70 \%$ ethanol, and stored in $70 \%$ ethanol until use. The BEHMs were prepared using a graded ethanol process with an automated tissue processor (Shandon Hypercenter XP; Thermo Electron, Waltham, MA) for 7 hours and 36 minutes. The BEHMs were then paraffin embedded. The paraffin-embedded samples were sectioned at $7 \mu \mathrm{m}$ on a microtome processor and placed on ProbeOn Plus slides (Fisher Scientific, Pittsburgh, PA) for histology. Hematoxylinand-eosin staining was used for morphologic analysis. Sections were viewed and photographed using a Nikon Axiophot inverted phase contrast microscope.

\section{Statistical analysis}

We used one-way analysis of variance (ANOVA) for all pair-wise comparisons. Minitab V13.31 (State College, PA) was used for statistical analysis with $p<0.05$.

\section{RESULTS}

Each bioreactor allowed up to 11 individual tissue specimens to be subjected as a group to the same mechanical stimulation protocol. Rapid manufacturing of the bioreactor systems using cell culture incubator tolerant materials as well as the use of standard cell culture disposable plastic in the bioreactor system design provided several significant advantages. Designs could be rapidly and inexpensively implemented, and design changes were readily made. Component interchangeability was maintained, and it was inexpensive to expand the capability to process fairly large numbers of tissue specimens simultaneously by simply rapid manufacturing additional bioreactor systems to meet the experimental need.

The design of the bioreactor proved suitable for transforming the static-engineered muscle culture for dynamic mechanical-loading studies without physical transfer of the construct. We were able to use the culture methodology developed in our laboratory and then place the $35 \mathrm{~mm}$ plates into the bioreactor for different mechanical-loading studies. In addition, using the microchip in the bioreactor, we were able to reprogram the training protocols fairly easily. 

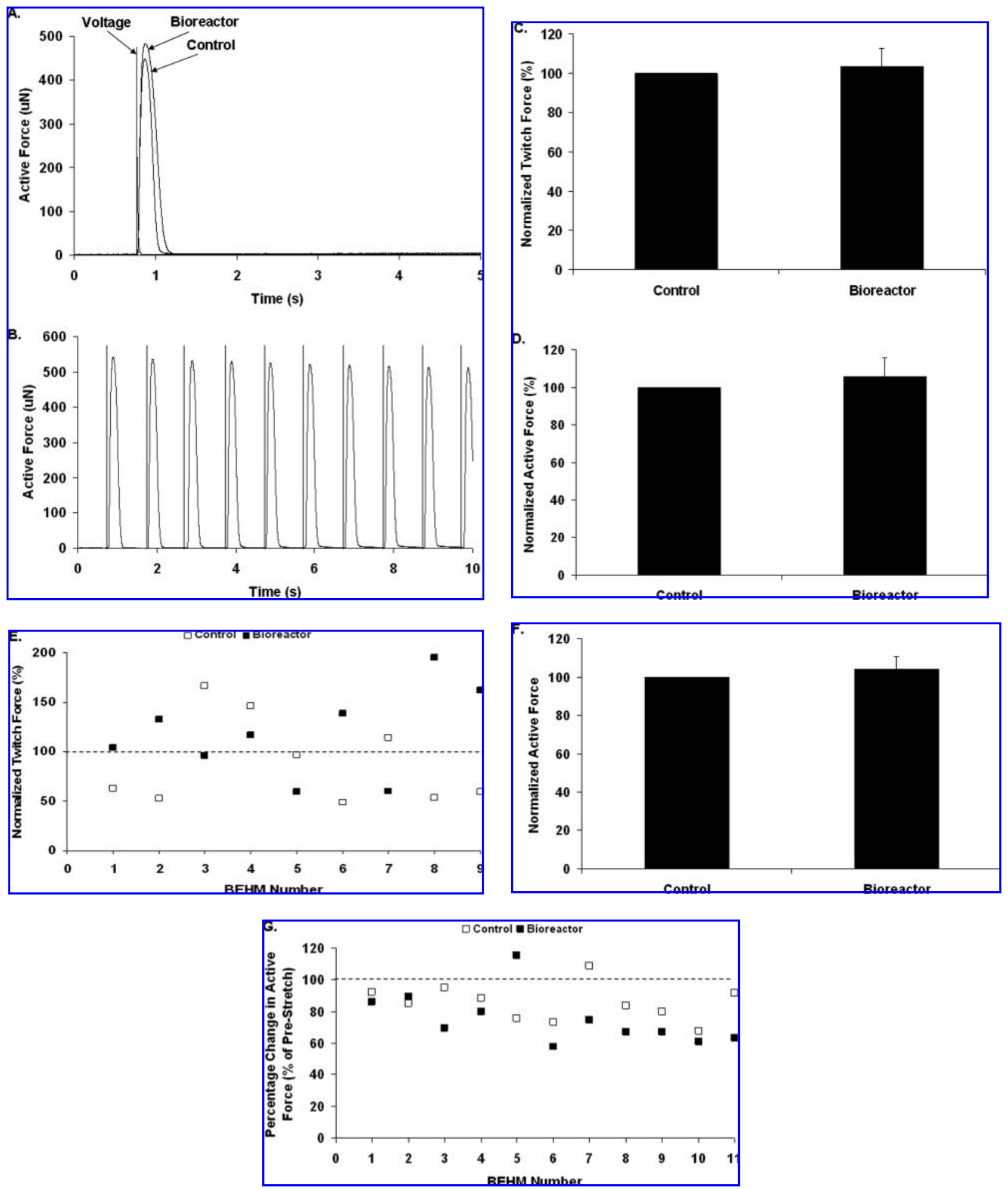

FIG. 5. Effect of controlled stretch on the active force of bioengineered heart muscles (BEHMs). BEHMs were placed in the bioreactor and subjected to a $10 \%$ stretch for 2, 6, and 24 hours per day for 7 days at a frequency of $1 \mathrm{~Hz}$. The maximum active force and the pacing characteristics of the BEHMs were evaluated after 7 days. (A) Representative force tracing of BEHMs in response to loading when stretched for 2 hours per day. (B) Pacing of the mechanically loaded BEHMs after a 2-hour loading protocol. There was no difference in the maximum active force of the BEHMs in response to loading protocols for (C) 2, (D) 6, and (F) 24 hours per day when compared to controls. Changes in the active force of individual BEHMs are displayed for the (E) 6-hour and (G) 24-hour loading protocol. The active force obtained after the loading protocol is normalized to the active force of the BEHM prior to starting the loading protocol. There was no statistically significant change in the active force or the specific force of the BEHMs in response to mechanical loading for the 2-, 4-, and 24-hour loading protocol. 
Our methodology for the formation of tissue-engineered constructs was easily reproducible. We engineered a total of 62 constructs for this study with zero incidence of failure for the controls, as evaluated by the ability of the isolated cells to remodel to form functional constructs. The mean active force for the controls, evaluated 14 days after initial cell plating, was found to be $12.1 \pm 0.62 \mathrm{kPa}(n=31)$ with a mean diameter of $207 \pm 5.7 \mu \mathrm{m}(n=31)$.

We were able to validate compatibility of the bioreactor system with our tissue-engineered constructs. During our 7day stretch protocol, there was no significant change in the diameter with the mean diameter of the constructs found to be $212 \pm 8.4 \mu \mathrm{m}(n=31)$. Of the 31 constructs that we tested in our bioreactor, we had 2 cases of failure, after being subjected to a stretch protocol of 6 hours loading during 24hour time points for 7 days. The maximum specific force of both constructs was in excess of the mean value of $12.1 \mathrm{kPa}$ prior to the loading protocol. There were no visible signs of construct damage and/or physical tearing of the two constructs. There was however a significant decrease in the diameter of these two constructs during the 7-day loading protocol, from 200 to $50 \mu \mathrm{m}$ for one construct and from 220 to $150 \mu \mathrm{m}$ for the second construct.

Our studies were designed to evaluate the stability of the BEHMs in response to mechanical stretch and we therefore selected mild loading conditions (Fig. 5). We found that there was no apparent physical damage of the BEHMs in response to varying stretch times of 2, 6, and 24 hours per 24-hour time period (Fig. 5A). We also found that there was no decline in the active force in response to these stretch protocols (Fig. 5C, D, F). The mean active force and the mean specific force of the constructs followed the same trend, as there was no significant change in construct diameter during stretch (data not shown). The mean specific force of BEHMs (both controls and stretched) loaded for 24 hours was lower than BEHMs stretched for 2 and 6 hours. These numbers reflect the batch variability in the primary cardiac cells, which were obtained from different batches for each of the three runs, rather than a response to the mechanicalloading protocols. We were also able to electrically pace the constructs (stretch and controls) after the three loading protocols (Fig. 5B) at frequencies of $1-7 \mathrm{~Hz}$ with no differences between the groups. The normalized active force (poststretch active force normalized to prestretch values) of the stretched BEHMs followed the same trend as the normalized active force for control BEHMs (Fig. 5E, G).

We evaluated the morphological characteristics of the constructs in response to the 6-hour cyclic loading protocol (Fig. 6). There was no physical damage to the constructs in response to the cyclic loading protocols. We found that the diameter of the constructs did not change significantly after the loading protocol and was in the order of 200-250 $\mu \mathrm{m}$ for both groups. There were a large number of well-aligned cells present throughout the thickness of the construct. In addition, we found large amounts of undegraded fibrin (which stains pink) in both groups with minimal damage to the

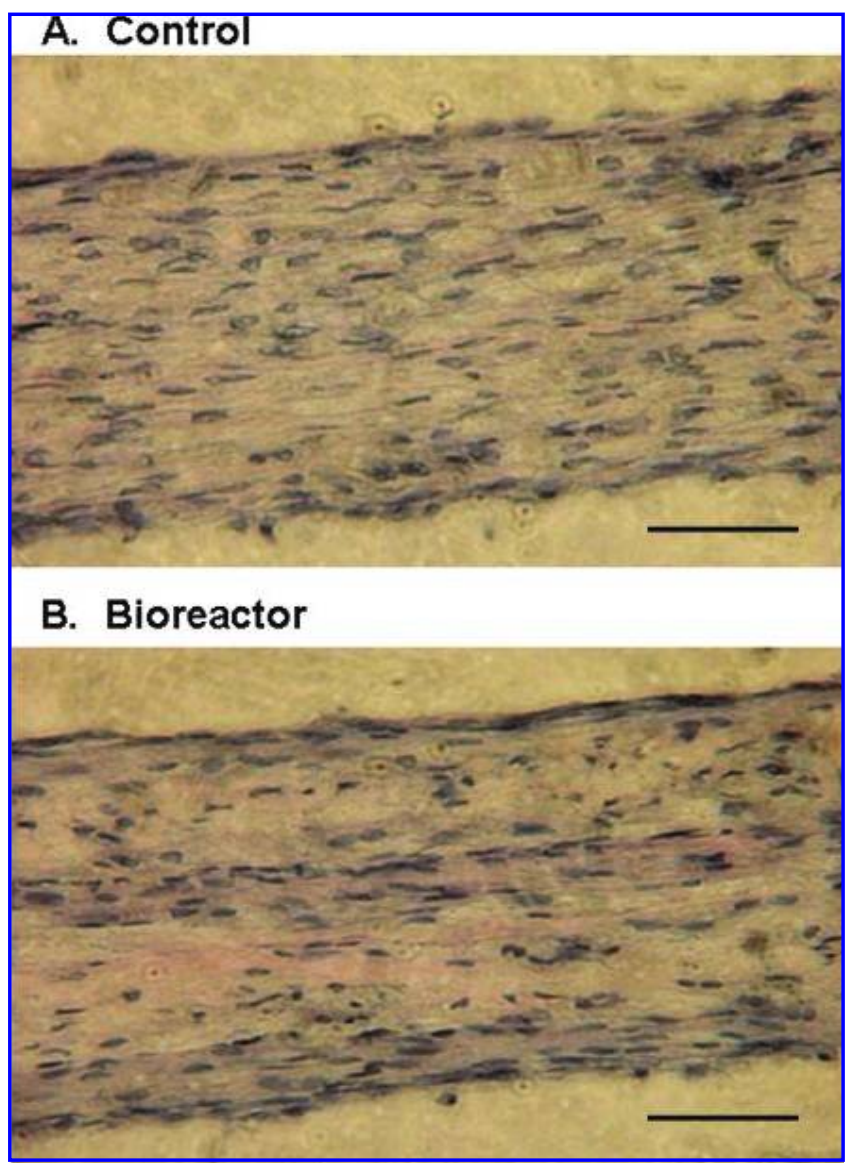

FIG. 6. Histological evaluation of constructs after cyclic loading. Hematoxylin-and-eosin staining was used to evaluate the morphological characteristics of the bioengineered heart muscles (BEHMs). (A) Control and (B) BEHMs after stretch. For these particular images, the BEHMs were stretched in the bioreactor for 6 hours every 24 hours for 7 days at a frequency of $1 \mathrm{~Hz}$. Scale bars represent $50 \mu \mathrm{m}$. Color images available online at www .liebertpub.com/ten.

fibers themselves. Our preliminary results showed a higher degree of undegraded fibrin in the mechanically stretched BEHMs (Fig. 6). These preliminary studies showed the compatibility of the bioreactor system with BEHMs as well as structural stability of the constructs in response to stretch protocols.

\section{DISCUSSION}

Our previous studies have been focused on defining conditions that promote the remodeling of isolated primary cardiac cells to form functional heart muscle under controlled in vitro cell culture conditions. Using our current methodology, we have been able to engineer three-dimensional heart muscle that exhibits several physiological metrics of cardiac function. ${ }^{11} \mathrm{We}$ have shown that the constructs generate active force upon electrical stimulation, can be electri- 
cally paced, show characteristics length-force and calcium sensitivity curves, and are responsive to cardio-active drugs. As we explore new ways to match the functional performance of our constructs to that of native cardiac muscle, we seek to explore the effects of mechanical stimulation on the functional outcome of the constructs. This particular study was designed to develop a bioreactor system to study the effect of controlled mechanical loading on the functional performance of three-dimensional heart muscle.

One of the critical technological hurdles in delivering controlled mechanical loading to tissue-engineered constructs is the lack of cell culture instrumentation. Commercially available systems are sparse and often not compatible with threedimensional constructs. Therefore, we sought to develop our own system to deliver controlled mechanical-loading protocols to our heart muscle constructs. The system we describe in this report was developed to be compatible with many of the self-organizing functional mammalian tissue constructs we have reported earlier, such as cardiac, ${ }^{17,18}$ skeletal, ${ }^{7,9,16,19}$ and smooth muscles, ${ }^{20}$ in addition to tendon and ligament, ${ }^{21}$ as well as recellularized peripheral nerve. ${ }^{22,23}$ The use of rapid manufacturing technology, local embedded control, standard disposable polystyrene cell culture Petri dishes, and interchangeable standard mechanical drive components enables the reconfiguration of each system to accommodate the tissues being studied in each case. The architecture of the system also allows the addition of expanded functionality, such as the addition of electrical stimulators for the tissues, the use of different-sized culture dishes, or the addition of force transducers to monitor the mechanical stiffness or active contractility of the engineered tissues. For this first study, only mechanical strain was applied.

Our experiments were designed to evaluate the compatibility of the bioreactors with our cardiac constructs, BEHMs. One of the challenges was to load the constructs onto the bioreactor platform without any physical damage to the constructs. Physical transfer of the constructs from the culture vessel to the bioreactor was eliminated by fabricating individual slots to allow placement of the entire culture vessel onto the bioreactor platform. Utilizing this methodology, we were able to load the constructs onto the bioreactor under sterile conditions without any physical damage.

Our second challenge was to apply controlled strain without physical damage to the BEHMs, either at the musclesuture interface or within the construct itself. We decided to load the BEHMs in a stepwise manner, starting with a loading protocol consisting of 2 hour of $10 \%$ stretch at $1 \mathrm{~Hz}$ per day for a period of 7 days. We found that there was no physical damage (evaluated by specific force) to the BEHMs with this relatively mild loading protocol and incrementally increased the time to 6 hours and subsequently 24 hours per day for 7 days. Of the 33 constructs that we subjected to loading protocol, only 2 constructs failed. There was a significant decrease in the diameter of these two BEHMs, indicative of rapid fibrin degradation thereby limiting functional remodeling. We have observed this behavior before and believe that the failure is a physiological response rather than a mechanical failure due to the loading protocol.

Histological evaluation supported structural integrity of the BEHMs in response to loading protocols. There appeared to be a greater fibrin content of the BEHMs subjected to the stretch protocols. In addition, we also found similar results for collagen type I, as determined by Mason's trichrone staining (data not shown), greater abundance of aligned collagen fibers for the BEHMs in response to mechanical stretch. We did not conduct a full histological analysis and can therefore only speculate that the changes in extracellular matrix are due to the mechanical stretch.

During our initial experiments, we were able to validate the compatibility of the bioreactor system with our constructs using gentle loading protocols. As we gradually increased the loading time from 2 to 24 hours, we found that there was no increase in contractile performance of the constructs. Although this was indicative of no mechanical damage to the constructs, there was some concern about the lack of functional improvement.

There has been one other study evaluating the effect of controlled mechanical stretch on the contractile performance of tissue-engineered heart muscle. ${ }^{13}$ A 6-day loading protocol at a frequency of $1.5 \mathrm{~Hz}$ and a strain of $5 \%$ resulted in a fourfold increase in force generation. The increase in force production was explained in terms of the hypertropic response of the cardiac myocytes. In the work described by Akhyari et al., ${ }^{12}$ the contractile performance was not evaluated; therefore, a comparison to this work proves difficult. Although we were not able to show an improvement in contractile force in response to mechanical stretch, we do believe that optimization of the stretch protocol will allow us to see functional improvements.

We believe that this study serves a twofold purpose. First, we describe a novel bioreactor system, which was rapid manufactured to create several systems for use in this and other ongoing studies to deliver controlled cyclic loading to tissue-engineered constructs. Second, we demonstrate compatibility of the bioreactor with our BEHM model.

\section{ACKNOWLEDGMENTS}

This work was supported by internal funds from the Section of Cardiac Surgery at the University of Michigan, Ann Arbor. Bioreactor development was supported by a contract from the Defense Advanced Research Projects Agency (DARPA) to R.G. Dennis (Award Number FA955005-1-0015).

\section{REFERENCES}

1. Eschenhagen, T., Fink, C., Remmers, U., Scholz, H., Wattchow, J., Weil, J., Zimmermann, W., Dohmen, H.H., Schafer, H., Bishopric, N., Wakatsuki, T., and Elson, E.L. Three-dimensional 
reconstitution of embryonic cardiomyocytes in a collagen matrix: a new heart muscle model system. FASEB J 11, 683, 1997.

2. Zimmermann, W.H., Fink, C., Kralisch, D., Remmers, U., Weil, J., and Eschenhagen, T. Three-dimensional engineered heart tissue from neonatal rat cardiac myocytes. Biotechnol Bioeng 68, 106, 2000.

3. Carrier, R.L., Papadaki, M., Rupnick, M., Schoen, F.J., Bursac, N., Langer, R., Freed, L.E., and Vunjak-Novakovic, G. Cardiac tissue engineering: cell seeding, cultivation parameters, and tissue construct characterization. Biotechnol Bioeng 64, 580, 1999.

4. Okano, T., Yamada, N., Sakai, H., and Sakurai, Y. A novel recovery system for cultured cells using plasma-treated polystyrene dishes grafted with poly(N-isopropylacrylamide). J Biomed Mater Res 27, 1243, 1993.

5. Shimizu, T., Yamato, M., Kikuchi, A., and Okano, T. Twodimensional manipulation of cardiac myocyte sheets utilizing temperature-responsive culture dishes augments the pulsatile amplitude. Tissue Eng 7, 141, 2001.

6. Akins, R.E., Boyce, R.A., Madonna, M.L., Schroedl, N.A., Gonda, S.R., McLaughlin, T.A., and Hartzell, C.R. Cardiac organogenesis in vitro: reestablishment of three-dimensional tissue architecture by dissociated neonatal rat ventricular cells. Tissue Eng 5, 103, 1999.

7. Dennis, R.G., and Kosnik, P.E. Excitability and isometric contractile properties of mammalian skeletal muscle constructs engineered in vitro. In Vitro Cell Dev Biol Anim 36, 327, 2000.

8. Dennis, R.G., Kosnik, P.E., Gilbert, M.E., and Faulkner, J.A. Excitability and contractility of skeletal muscle engineered from primary cultures and cell lines. Am J Physiol Cell Physiol 280, C288, 2001.

9. Kosnik, P.E., Faulkner, J.A., and Dennis, R.G. Functional development of engineered skeletal muscle from adult and neonatal rats. Tissue Eng 7, 573, 2001.

10. Baar, K., Birla, R., Boluyt, M.O., Borschel, G.H., Arruda, E.M., and Dennis, R.G. Heart muscle by design: self-organization of rat cardiac cells into contractile 3-D cardiac tissue. FASEB J 19, 275, 2005.

11. Huang, Y.C., Khait, L., and Birla, R.K. Contractile threedimensional bioengineered heart muscle for myocardial regeneration. J Biomed Mater Res A 80, 719, 2007.

12. Akhyari, P., Fedak, P.W., Weisel, R.D., Lee, T.Y., Verma, S., Mickle, D.A., and Li, R.K. Mechanical stretch regimen enhances the formation of bioengineered autologous cardiac muscle grafts. Circulation 106 Suppl, I137, 2002.

13. Fink, C., Ergun, S., Kralisch, D., Remmers, U., Weil, J., and Eschenhagen, T. Chronic stretch of engineered heart tissue induces hypertrophy and functional improvement. FASEB J 14, 669, 2000.
14. Dennis, R.G., Dow, D.E., and Faulkner, J.A. An implantable device for stimulation of denervated muscles in rats. Med Eng Phys 25, 239, 2003.

15. Boluyt, M.O., Zheng, J.S., Younes, A., Long, X., O’Neill, L., Silverman, H., Lakatta, E.G., and Crow, M.T. Rapamycin inhibits alpha 1-adrenergic receptor-stimulated cardiac myocyte hypertrophy but not activation of hypertrophy-associated genes. Evidence for involvement of p70 S6 kinase. Circ Res 81, 176, 1997.

16. Huang, Y.C., Dennis, R.G., Larkin, L., and Baar, K. Rapid formation of functional muscle in vitro using fibrin gels. J Appl Physiol 98, 706, 2005.

17. Baar, K., Birla, R., Boluyt, M.O., Borschel, G.H., Arruda, E.M., and Dennis, R.G. Self-organization of rat cardiac cells into contractile 3-D cardiac tissue. FASEB J 19, 275, 2005.

18. Birla, R.K., Borschel, G.H., Dennis, R.G., and Brown, D.L. Myocardial engineering in vivo: formation and characterization of contractile, vascularized three-dimensional cardiac tissue. Tissue Eng 11, 803, 2005.

19. Kosnik, P.E., and Dennis, R.G. Mesenchymal cell culture: functional mammalian skeletal muscle constructs. In: Atala, A., Lanza, R., eds. Methods in Tissue Engineering. San Diego: Harcourt, Academic Press, 2002, pp. 299-306.

20. Hecker, L., Baar, K., Dennis, R.G., and Bitar, K.N. Development of a three-dimensional physiological model of the internal anal sphincter bioengineered in vitro from isolated smooth muscle cells. Am J Physiol Gastrointest Liver Physiol 289, G188, 2005.

21. Calve, S., Dennis, R.G., Kosnik, P.E., Baar, K., Grosh, K., and Arruda, E.M. Engineering of functional tendon. Tissue Eng 10, 755, 2004.

22. Borschel, G.H., Kia, K.F., Kuzon, W.M., Jr., and Dennis, R.G. Mechanical properties of acellular peripheral nerve. J Surg Res 114, 133, 2003.

23. Haase, S.C., Rovak, J.M., Dennis, R.G., Kuzon, W.M., Jr., and Cederna, P.S. Recovery of muscle contractile function following nerve gap repair with chemically acellularized peripheral nerve grafts. J Reconstr Microsurg 19, 241, 2003.

Address reprint requests to: Ravi K. Birla, Ph.D.

Section of Cardiac Surgery University of Michigan

MSRB II, B560, 1150 West Medical Center Drive Ann Arbor, MI 48109

E-mail: rbirla@umich.edu 
This article has been cited by:

1. R. K. Birla, Y. C. Huang, R. G. Dennis. 2008. Effect of streptomycin on the active force of bioengineered heart muscle in response to controlled stretch. In Vitro Cellular \& Developmental Biology - Animal 44:7, 253-260. [CrossRef]

2. Louise Hecker, Luda Khait, Desmond Radnoti, Ravi Birla. 2008. Development of a Microperfusion System for the Culture of Bioengineered Heart Muscle. ASAIO Journal 54:3, 284-294. [CrossRef] 